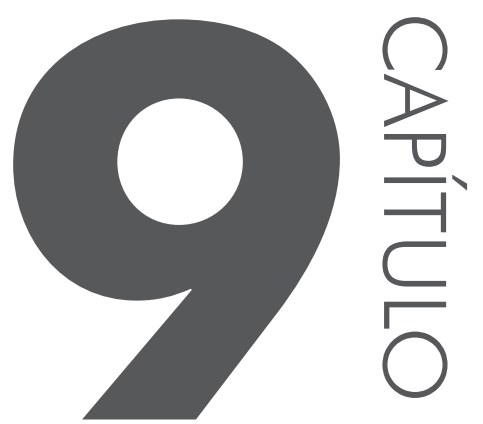

\title{
TURBINA EÓLICA DE EFEITO MAGNUS: MODELAGEM, PROTÓTIPOS E MPPT
}

Maro Jinbo

Instituto Federal de Santa

Catarina, Departamento de Engenharia de Controle e Automação. maro@ifsc.edu.br

Daniel Senter Instituto Federal de Santa Catarina, Departamento de Engenharia de Controle e Automação. danielsenter1@gmail.com

\author{
Felix Alberto Farret \\ Universidade Federal de Santa \\ Maria, Departamento de Engenharia \\ Elétrica. \\ fafarret@gmail.com
}

Ghendy Cardoso Junior Universidade Federal de Santa Maria, Departamento de Engenharia Elétrica. ghendy@ufsm.br

Edson Ribeiro dos Santos Universidade Federal da Fronteira Sul, Departamento de Matemática. edson.santos@uffs.edu.br

\section{RESUMO}

Este artigo apresenta a modelagem matemática de turbinas eólicas que não utilizam pás convencionais para captar a energia cinética dos ventos. No lugar das pás, a 
turbina possui cilindros girantes acionados por um ou mais motores elétricos. Cilindros girantes imersos no ar criam diferenças de pressões originando forças (de sustentação) transversais à direção do fluxo de ar. Esse fenômeno físico é conhecido como Efeito Magnus. Essas forças aplicadas aos cilindros girantes de uma turbina proporcionam o torque no eixo principal, que é transmitida ao gerador elétrico. Simulando-se com o modelo matemático que descreve o comportamento da turbina Magnus, demonstram-se os critérios necessários para o MPPT (Maximum Power Point Tracking).

Palavras-chave: Turbina Eólica, Efeito Magnus, Cilindros Girantes, MPPT.

\section{INTRODUC̣ÃO}

A turbina eólica de efeito Magnus possui cilindros girantes no lugar das pás convencionais, conforme a Figura 1, que apresenta um protótipo de $1 \mathrm{~m}$ de diâmetro usinado em poliacetal. Esses cilindros podem ser acionados por um motor elétrico (coroa no eixo do motor e pinhões em cada um dos cilindros) no caso do protótipo da Figura 1 ou mais motores elétricos (individualmente para cada um dos cilindros). As velocidades angulares (rotação) $\Omega_{1}$ e $\Omega_{2}$ são do rotor da turbina e dos cilindros respectivamente. Seu funcionamento baseia-se no princípio físico conhecido por Efeito Magnus.

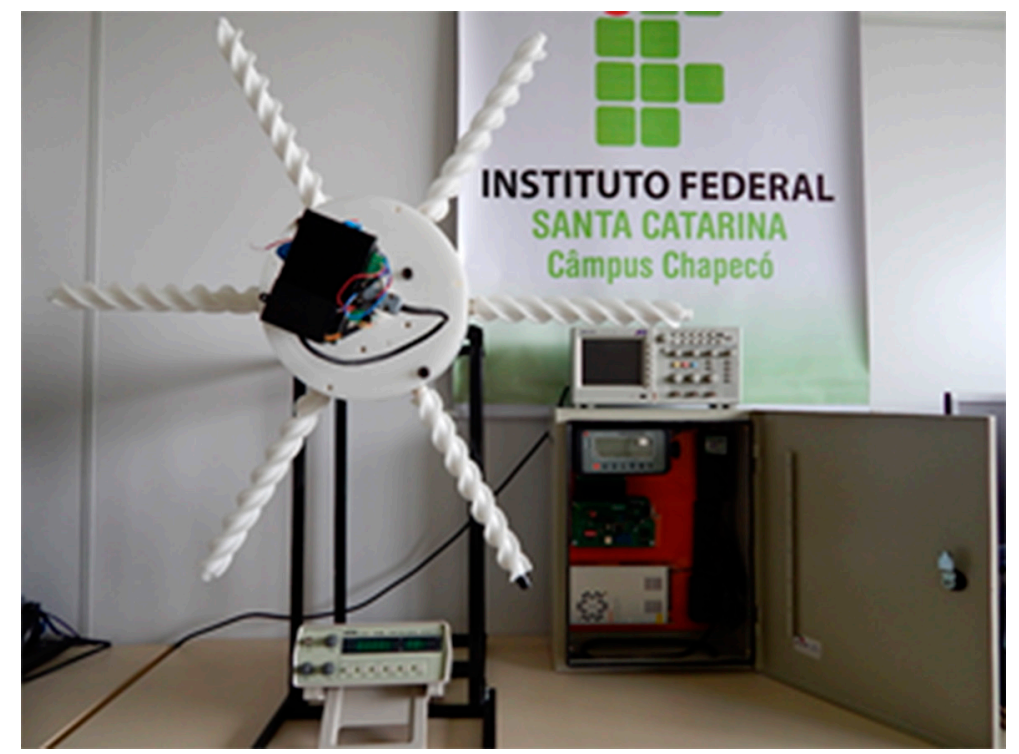

Figura 1 Protótipo de turbina eólica Magnus de $1 \mathrm{~m}$ de diâmetro.

Os registros dos primeiros protótipos de turbinas eólicas de efeito Magnus não são recentes (VAUGHN, 2009). Em 1926, Flettner construiu uma turbina eólica de eixo horizontal com quatro cilindros cônicos acionados por um motor 
elétrico. Os cilindros tinham $5 \mathrm{~m}$ de comprimento e $0,8 \mathrm{~m}$ de diâmetro no ponto médio. O rotor tinha $20 \mathrm{~m}$ de diâmetro apoiado em uma torre de $33 \mathrm{~m}$, com uma faixa de potência de $30 \mathrm{KW}$ para uma velocidade de vento de $10 \mathrm{~m} / \mathrm{s}$.

Em 1983, um empresário da Califórnia construiu uma turbina Magnus que foi denominada de Barrel-blade (pás de barril) com $17 \mathrm{~m}$ de diâmetro, com capacidade suposta de $110 \mathrm{~kW}$. O aerogerador foi mais tarde transferido para San Gorgonio Pass, no sul da Califórnia, para testes ao vento.

Recentemente, em 2007, uma empresa japonesa, por meio de esforços em conjunto entre indústria, governo e universidades, desenvolveu e lançou comercialmente uma turbina eólica de efeito Magnus (MECARO, 2007). Os cilindros dessa turbina Magnus possuem duas características relevantes, uma barbatana em espiral e uma tampa na extremidade, que aumentam o efeito Magnus, proporcionando maior torque (MURAKAMI, 2009).

$\mathrm{Na}$ Rússia, realizaram-se experimentos com protótipos de turbinas eólicas Magnus em um túnel de vento. Comprovou-se que a turbina Magnus, mesmo em velocidades de vento de $2 \mathrm{~m} / \mathrm{s}$, apresenta um coeficiente de potência suficiente para gerar potência útil e aproveitar essas baixas velocidades de vento. A turbina pode ser operada também para velocidades maiores que 35 a $40 \mathrm{~m} / \mathrm{s}$ de vento, bem superiores ao limite das turbinas convencionais (BYCHKOV, 2007), (BYCHKOV E DOVGA, 2008), (BYCHKOV, 2008).

$\mathrm{Na}$ Figura 2, tem-se um cilindro de raio $\mathrm{R}$ rotacionando com velocidade angular $\Omega$, imerso em um fluido de densidade $\rho$, cuja velocidade laminar é $\mathrm{U}_{\infty}$. Teoricamente, o cilindro está sujeito a uma força resultante na direção perpendicular ao fluxo do fluido, denominada força de sustentação $\mathrm{F}_{\mathrm{Y}}$ (lift force), e de arrasto $\mathrm{F}_{\mathrm{X}}$ (drag force), fenômeno conhecido como Efeito Magnus (MUNSON, 2009), (WHITE, 2012). Em teoria, a força de arrasto $F_{X}$ é nula, mas experimentalmente comprova-se que esse valor não é nulo e elava-se com o aumento da velocidade relativa da superfície do cilindro (WHITE, 2012).

Imai e Kato (2010) realizaram simulações numéricas (Computational Fluid Dynamics - CFD) de um cilindro com seis barbatanas em espiral conforme a disposição da seção transversal apresentada na Figura 3(a) e compararam com um cilindro liso. No cilindro liso da Figura 3(b) e no cilindro com barbatana da Figura 3(c), atuam as forças de sustentação na vertical $\mathrm{F}_{\mathrm{Y}}$ ( lift force) decorrente do efeito Magnus (de cima para baixo) e das forças de arrasto na horizontal $\mathrm{F}_{\mathrm{x}}$ (drag force) (da esquerda para direita) a que estão sujeitos os cilindros girantes de raio $\mathrm{R}$ imersos em um fluxo de ar de densidade $\rho$. 


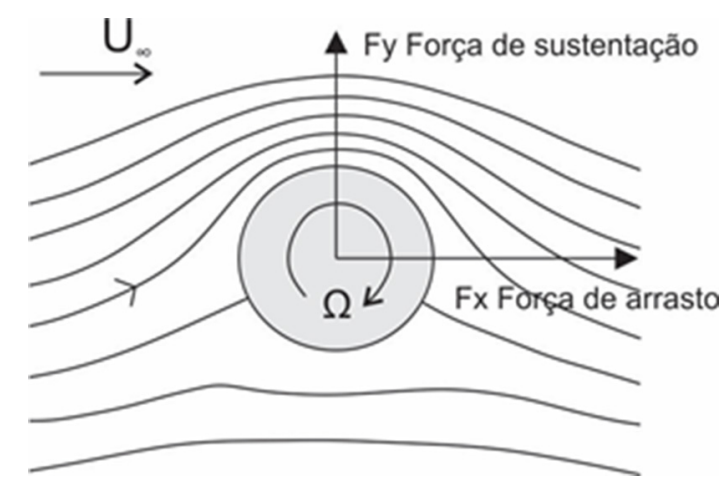

Figura 2 Cilindro rotacionando imerso em um fluido.

Fonte: Burton et al. (2011).

A velocidade do vento laminar é identificada como $\mathrm{U}_{\infty}$ e $\Omega$ é a velocidade angular do cilindro. A relação entre $\Omega \cdot R / U_{\infty}$ é de 0,4 nas simulações realizadas por Imai e Kato. A Figura 3(b) apresenta o fluxo de ar ao redor do cilindro girante com superfície lisa de mesmo diâmetro do cilindro com barbatana em espiral.

Os pontos em destaques denominados "separation point", ou seja, "pontos de descolamentos" são regiões onde se inicia o descolamento do fluxo de ar da parede do cilindro. A Figura 3(c) apresenta o fluxo de ar ao redor do cilindro com barbatana em espiral, conforme a Figura 3(a). É possível verificar que o descolamento do fluxo de ar na face inferior do cilindro ocorre em um ponto posterior. Esse fato provoca uma superfície maior na face inferior do cilindro com pressão relativa menor que a face superior, portanto a força de sustentação na face superior é maior do que em um cilindro sem barbatanas.
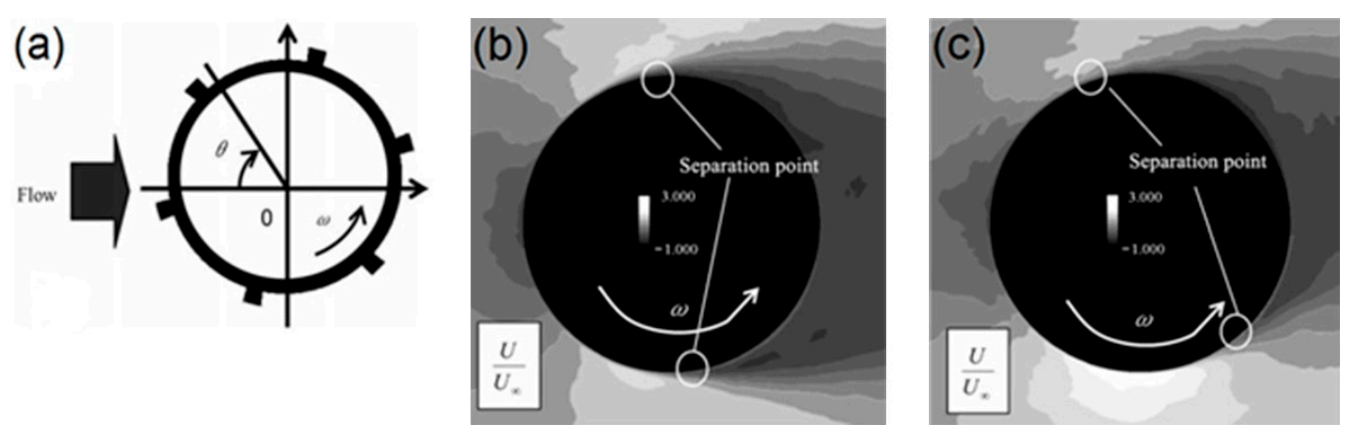

Figura 3 Simulação numérica CFD: (a) Seção transversal do cilindro com seis barbatanas em espiral. (b) Cilindro liso. (c) Cilindro com barbatanas em espiral.

Fonte: Imai e Kato (2010). 


\section{TURBINA EÓLICA DE EFEITO MAGNUS}

\subsection{A potência da turbina eólica Magnus}

A ilustração da Figura 4 representa uma turbina eólica de efeito Magnus com seis cilindros girantes, em que estão indicados alguns parâmetros importantes que são utilizados nas equações que modelam o seu comportamento.

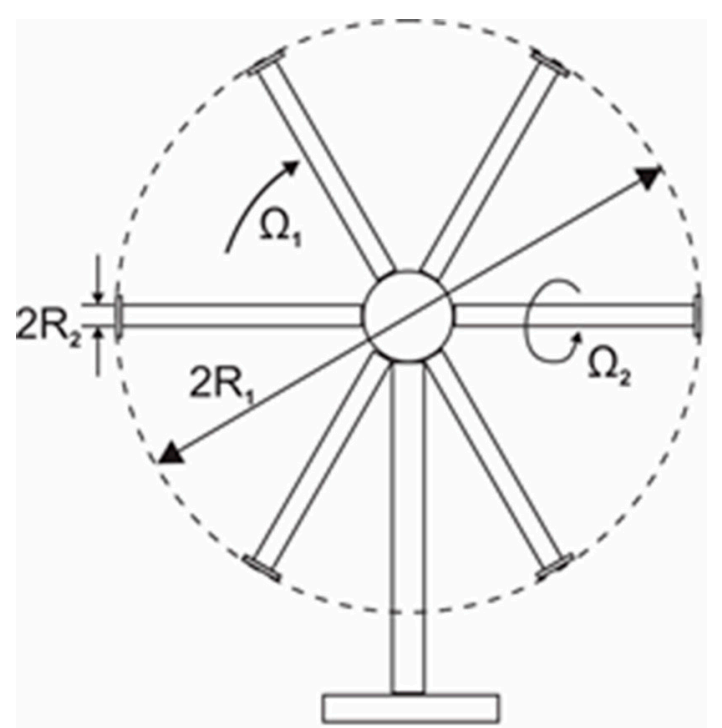

Figura 4 Turbina eólica de efeito Magnus.

Onde:

$\mathrm{N}$ = número de cilindros girantes;

$\mathrm{R}_{1}$ = raio da turbina $[\mathrm{m}]$;

$\Omega_{1}=$ velocidade angular da turbina $[\mathrm{rad} / \mathrm{s}]$;

$\mathrm{R}_{2}=$ raio dos cilindros $[\mathrm{m}]$;

$\Omega_{2}=$ velocidade angular dos cilindros $[\mathrm{rad} / \mathrm{s}]$;

$\mathrm{U}_{\infty}=$ velocidade laminar do vento $[\mathrm{m} / \mathrm{s}]$;

$\rho=$ densidade do ar $\left[\mathrm{kg} / \mathrm{m}^{3}\right]$.

A razão entre a velocidade tangencial da extremidade do cilindro da turbina e a velocidade do vento é conhecida como TSR (Tip Speed Ratio) da turbina Magnus, (SLOOTWEG, 2003). A TSR do rotor é dada pela Equação (1):

$$
\lambda_{1}=\frac{\Omega_{1} \cdot R_{1}}{U_{\infty}}
$$


A expressão da velocidade relativa (TST) do cilindro em relação à velocidade do vento é definida pela Equação (2) (LUO, 2011), (SUN, 2012):

$$
\lambda_{2}=\frac{\Omega_{2} \cdot R_{2}}{U_{\infty}}
$$

Portanto, a potência mecânica extraída do vento pela turbina Magnus é representada pela Equação (3) (BURTON, 2011), (SLOOTWEG, 2003), (LUO, 2011), (SUN, 2012), (MONROY, 2006):

$$
P=0.5 \cdot \rho \cdot U_{\infty}^{3} \cdot \pi \cdot R_{1}^{2} \cdot C_{P}
$$

A Equação (4) analítica é referente ao coeficiente de potência $C_{P}$ da turbina de efeito Magnus e foi obtida com base nos princípios da dinâmica dos fluidos e da teoria da quantidade de movimento do elemento pá (Blade Element Momentum Theory - BEM) desprezando-se a influência de outros fatores relevantes, como força de arrasto, perdas nas extremidades dos cilindros, interação entre os cilindros e efeito do diâmetro do cubo na potência de saída da turbina. O modelo é melhorado com valores de $\mathrm{C}_{\mathrm{p}}$ obtidos experimentalmente, que representam mais proximamente a realidade (LUO, 2011; SUN, 2012). O coeficiente de potência representa o percentual de potência que a turbina consegue extrair dos ventos. $\mathrm{O}$ valor limite do coeficiente de potência para turbinas eólicas de efeito Magnus é $\mathrm{C}_{\mathrm{p}}=0,593$.

$$
C_{P}=\frac{N \cdot \Omega_{1} \cdot \Omega_{2} \cdot R_{2}^{2}}{2 \cdot U_{\infty}^{2}} \cdot\left(1+\sqrt{1-\frac{N \cdot \Omega_{1} \cdot \Omega_{2} \cdot R_{2}^{2}}{U_{\infty}^{2}}}\right)
$$

\subsection{O MPPT da turbina eólica Magnus}

A potência mecânica $\mathrm{P}_{\text {mec }}(\mathrm{W})$ produzida pela turbina eólica Magnus, bem como as turbinas eólicas convencionais, é o produto entre o torque T [N.m] e a velocidade de rotação da turbina $\Omega_{1}[\mathrm{rads} / \mathrm{s}]$, de acordo com a Equação (5). Existe uma velocidade de rotação ótima que proporciona a máxima potência mecânica no eixo. Portanto, é necessário rastrear a velocidade de rotação ótima da turbina (MPPT) (HAN, 2009), (YANG, 2010), (DUAN, 2006), (RIBEIRO, 2013), (JINBO, 2015).

$$
P_{m e c}=T \cdot \Omega_{1}
$$

A Figura 5 representa uma curva típica da potência mecânica produzida pela turbina Magnus em função de $\lambda_{2}$ (velocidade de rotação relativa dos cilindros) para uma determinada $\lambda_{1}$ constante (TSR). Para cada uma das quatro velocidades de ventos ilustradas na Figura 5, traçou-se uma curva de potência mecânica em 
função de $\lambda_{2}$ utilizando-se da Equação (3) potência mecânica e da Equação (4) coeficiente de potência. Pode-se observar nas curvas de potência traçadas que existe valor de $\lambda_{2}$, ou seja, de velocidade dos cilindros, que resulta em um ponto de máximo. Portanto, o sistema deve rastrear essa rotação ótima dos cilindros que proporcione o valor máximo de potência.

Considerando-se que a turbina Magnus necessita de um motor elétrico ou mais para rotacionar os cilindros, o sistema de controle MPPT da rotação dos cilindros deve rastrear a máxima potência líquida $P_{\text {liq }}$, ou seja, a potência mecânica $\mathrm{P}_{\text {mec }}$ gerada menos a potência consumida $\mathrm{P}_{\text {cons }}$ pelo sistema de acionamento. Pode-se afirmar, portanto, que existe uma velocidade de vento mínima de partida da turbina para que se extraia potência líquida.

A extração da máxima potência elétrica de um sistema eólico Magnus implica rastrear a rotação ótima da turbina $\Omega_{1}$ e simultaneamente a rotação ótima dos cilindros $\Omega_{2}$.

$$
P_{\text {liq }}=P_{\text {mec }}-P_{\text {cons }}
$$

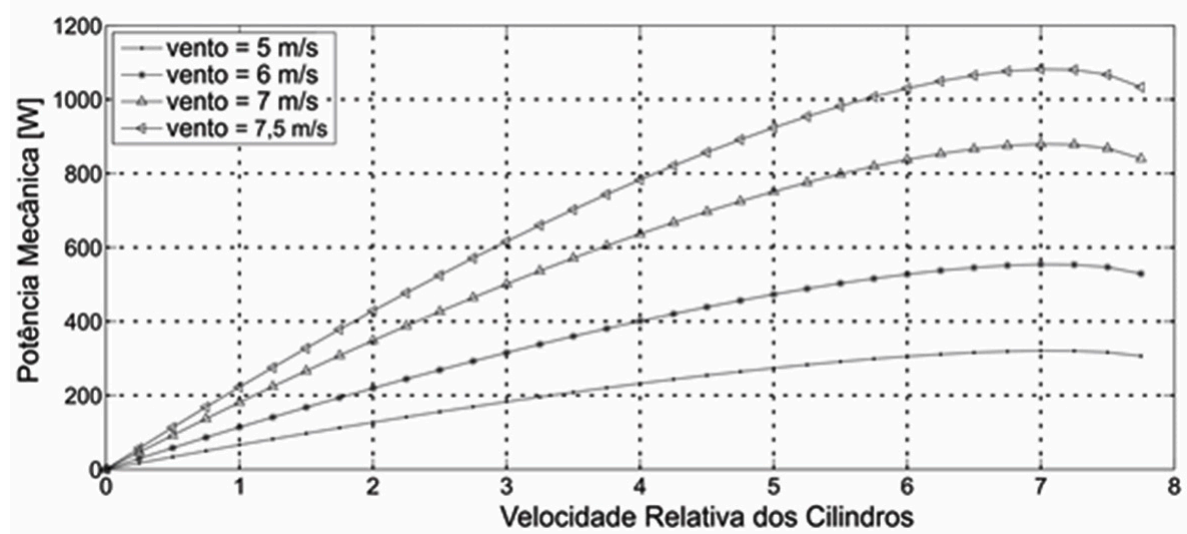

Figura 5 Potência mecânica vs. $\lambda_{2}$.

\section{AEROGERADOR MAGNUS PROPOSTO}

\subsection{Protótipos de turbinas Magnus}

A Figura 1 apresenta o protótipo de turbina Magnus de $1 \mathrm{~m}$ de diâmetro, fabricado em poliacetal com seis cilindros de $4 \mathrm{~cm}$ de diâmetro, com barbatanas em espiral com duas entradas. Os cilindros e o cubo da turbina foram usinados em um centro de usinagem $\mathrm{CNC}$ de quatro eixos. Na parte frontal do cubo (objeto preto), pode-se observar o servo drive e o motor brushless (sem escovas) que 
realizam o acionamento e o controle da velocidade de rotação dos cilindros. A transmissão da rotação do motor brushless para os cilindros é realizada através de uma coroa e um conjunto de pinhões dispostos um em cada extremidade interna de cada cilindro. Além do protótipo, na Figura 1, pode-se observar um quadro de comando contendo a placa microcontrolada de controle da turbina, fonte de alimentação e display do sensor de torque.

A Figura 6 consiste no protótipo de turbina Magnus de $3 \mathrm{~m}$ de diâmetro com seis cilindros de 10,5 cm de diâmetro. Esse protótipo foi fabricado em alumínio aeronáutico 7075, PVC, poliacetal e polietileno. Mas é significativamente diferente do protótipo de poliacetal de $1 \mathrm{~m}$ de diâmetro, pois foi projetado em outra concepção de turbina e não possui mancais no cubo para suportar os seis cilindros devido à existência de um eixo interno fixo ao cubo. Ao redor desses eixos é que rotacionam os seis cilindros de PVCs com barbatanas em espiral.

Nos dois protótipos de turbina Magnus que foram construídos, foi utilizado o mesmo sistema de acionamento dos cilindros (servo drive e motor brushless) (LEADSHINE, 2015) e o mesmo sistema de controle (placa com microcontrolador). No protótipo de $3 \mathrm{~m}$ de diâmetro, devido à necessidade de maior torque, foi fabricada e instalada uma caixa de engrenagens planetárias na extremidade do eixo do motor brushless.

O microcontrolador executa um algoritmo subida de colina (Hill Climbing Control - HCC) para realizar o MPPT do aerogerador Magnus.

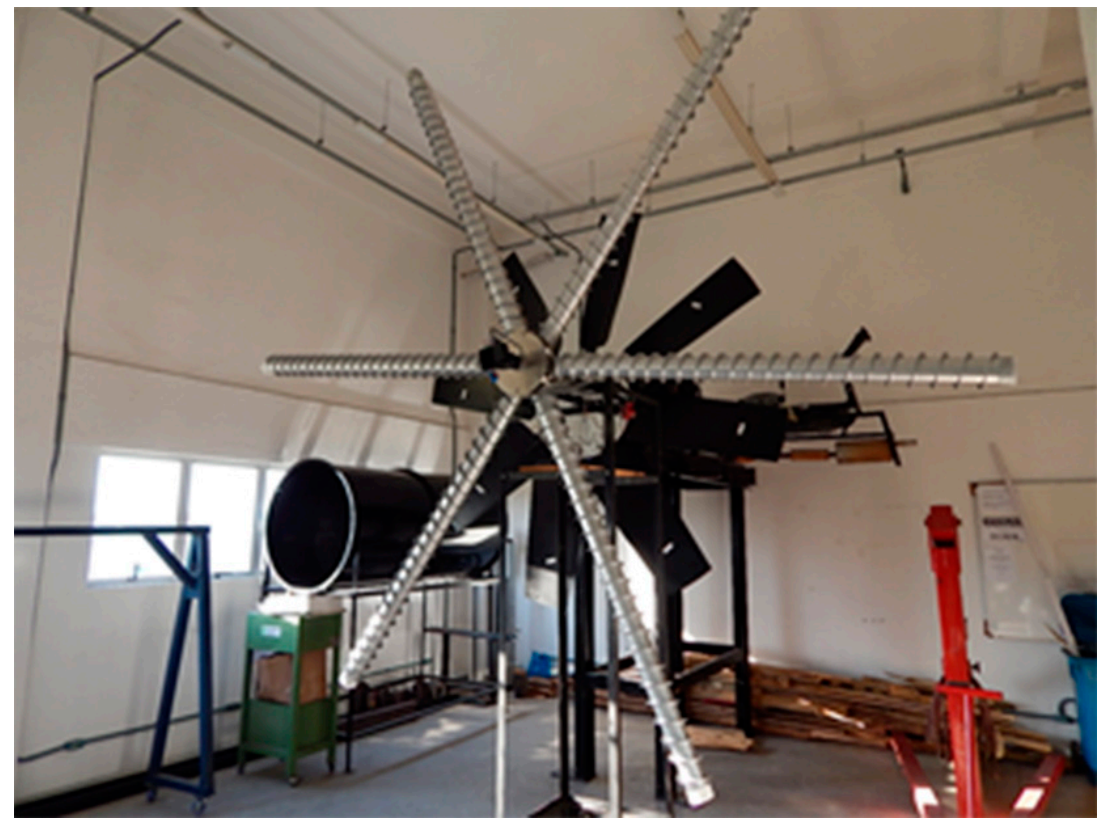

Figura 6 Turbina eólica de efeito Magnus de $3 \mathrm{~m}$ de diâmetro. 


\subsection{Sistema de MPPT do aerogerador Magnus proposto}

$\mathrm{Na}$ Figura 7, apresenta-se um diagrama de blocos simplificado de uma proposta de sistema eólico de efeito Magnus conectado à rede elétrica. O sistema é composto por turbina Magnus, servo drive e motor brushless, anel coletor especial, caixa de engrenagens, gerador de ímãs permanentes (Permanent Magnet Synchronous Generator - PMSG), retificador, conversor estático CC/CC tipo boost e inversor monofásico (on grid) conectado à rede elétrica.

O motor brushless, por não possuir escovas, reduz a periodicidade da manutenção em relação a um motor CC e, por ter uma boa controlabilidade através do servo drive, pode ser considerado uma boa escolha para esse tipo de aplicação.

$\mathrm{O}$ anel coletor é um tipo especial que possibilita a transmissão da alimentação e o sinal de controle para o rotor da turbina onde se localiza o motor brushless e o servo drive (MOFLON, 2015).

A tensão trifásica alternada na saída do gerador PMSG é retificada e depois elevada pelo conversor boost, adequando-se para os níveis de tensão da entrada do inversor conectado à rede elétrica. Esse é o fluxo da energia captada dos ventos.

Objetiva-se, por meio do diagrama de bloco da Figura 7, apresentar como e onde o controle MPPT deve atuar para se extrair a potência máxima dos ventos (JINBO, 2015).

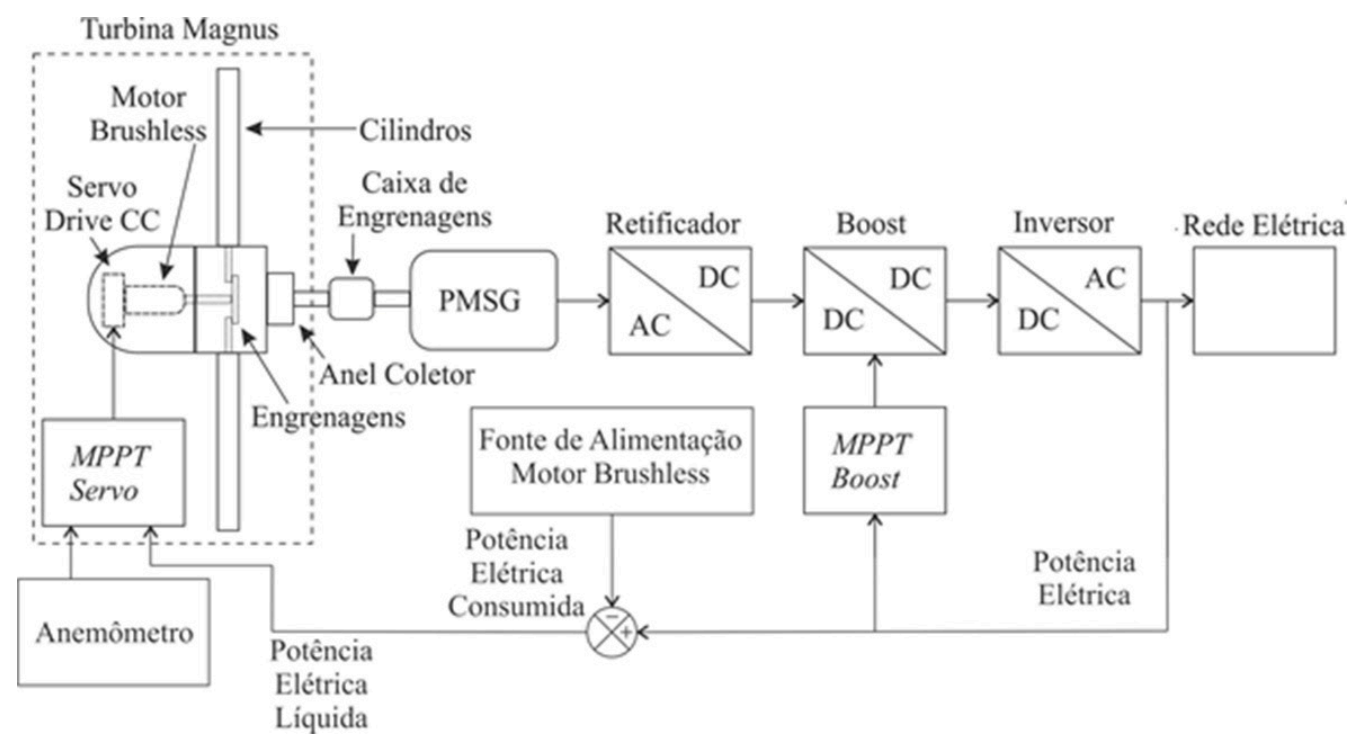

Figura 7 Proposta de sistema eólico Magnus para o MPPT.

O bloco "MPPT Servo" representa o controle realizado sobre o servo drive rastreando uma rotação ótima dos cilindros, que proporcione a máxima potência 
para cada velocidade de vento. Esse bloco utiliza como informação de entrada a potência elétrica líquida gerada $\mathrm{P}_{\mathrm{liq}}$ e a velocidade do vento proveniente do anemômetro. A potência elétrica líquida é a potência entrega à rede elétrica subtraindo-se a potência consumida $\mathrm{P}_{\text {cons }}$ pelo sistema de acionamento (servo drive e motor brushless). O "MPPT Servo" vai rastrear, portanto, a máxima potência elétrica líquida gerada ajustando-se a rotação dos cilindros da turbina Magnus. A leitura de velocidade do vento faz-se necessária para que a turbina Magnus permaneça desligada até que se atinja uma velocidade de vento mínima que posso gerar potência líquida positiva.

A placa de controle microcontrolada fica na parte estacionária do aerogerador juntamente com o PMSG e os conversores estáticos. Portanto, faz-se necessária a transmissão da energia da fonte de alimentação do motor brushless bem como o sinal de referência de controle para o servo drive.

O bloco "MPPT Boost" atua no controle do conversor estático CC/CC (boost) variando a razão cíclica para que seja transferida do gerador PMSG para a rede elétrica na máxima potência elétrica, o que acarretará também uma variação da velocidade de rotação do conjunto turbina e gerador.

Existem vários algoritmos que podem ser utilizados no MPPT, tanto do servo drive como do boost, tais como: HCC, PO Perturba e Observa, Lógica Fuzzy entre outros (RUSSEL, 2010), (SIMÕES e FARRET, 2004). Na Seção 3.3, será apresentado o algoritmo HCC utilizado nos protótipos.

\subsection{Algoritmo MPPT - HCC}

O algoritmo subida de colina (HCC) com passo fixo rastreia o ponto máximo (PM) da curva da potência líquida $P_{\text {liq }}$ através do incremento e decremento da tensão de referência $\mathrm{V}_{\text {ref }} \operatorname{com}$ passo fixo $\Delta \mathrm{V}_{\text {fixo }}$. $\mathrm{O}$ algoritmo está sempre buscando o PM como está apresentado na Figura 8.

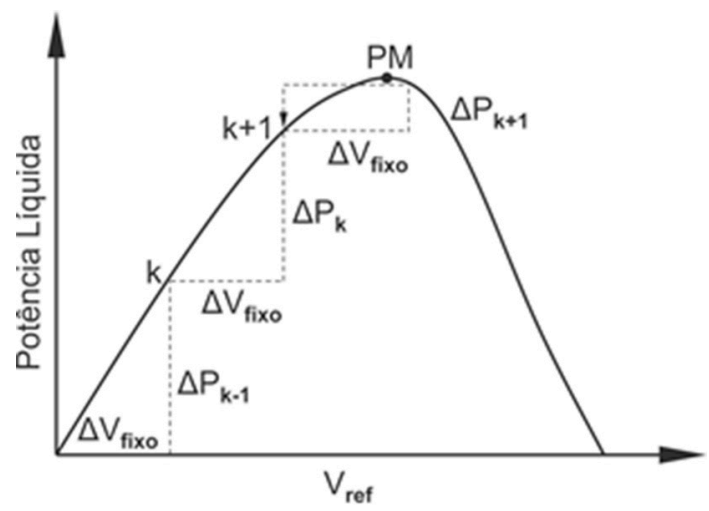

Figura 8 Algoritmo Hill Climbing com passo fixo. 
Um passo relativamente grande pode resultar em um tempo reduzido para atingir o topo da curva, mas pode resultar em maiores oscilações em torno do PM.

$\mathrm{O}$ algoritmo HCC com passo adaptativo realiza o rastreamento do ponto máximo (PM) sobre a curva da potência líquida $\mathrm{P}_{\text {liq }}$ através do incremento e decremento da tensão de referência $\mathrm{V}_{\text {ref }}$, Equação (7), com passo adaptativo $\Delta \mathrm{V}_{\text {adap }}$, Equação (8). O algoritmo sempre busca pelo PM como está apresentado na Figura 9 . $\mathrm{O}$ valor de $\Delta \mathrm{V}_{\text {adap }}$ vai diminuindo à medida que a potência vai se aproximando do PM e assim reduzem-se as oscilações em torno desse ponto. Na Equação (8), $\varepsilon$ é uma constante infinitesimal.

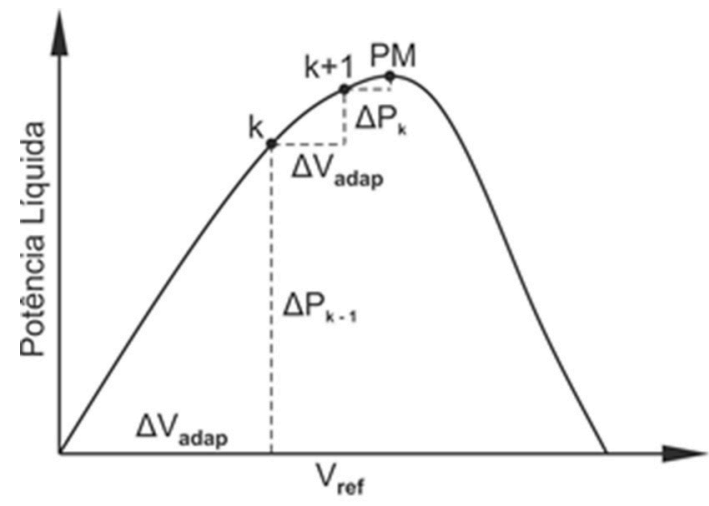

Figura 9 Algoritmo Hill Climbing com passo adaptativo.

$$
\begin{gathered}
\left(V_{\text {ref }}\right)_{k}=\left(V_{\text {ref }}\right)_{k-1} \pm \Delta V_{\text {adap }} \\
\Delta V_{\text {adap }}=\varepsilon \cdot \Delta P_{k} \cdot \Delta V_{k}
\end{gathered}
$$

A Figura 10 apresenta o fluxograma do algoritmo de controle dos cilindros girantes, corresponde ao bloco "MPPT Servo" da Figura 7. Inicialmente, é realizada a leitura da velocidade do vento, que é comparada com a velocidade mínima denotada por vento $_{\min }$. Para ventos abaixo desse valor, o cilindro girante permanece estático, evitando gastos desnecessários de energia. Quando a velocidade do vento está acima desse valor, o sistema gera potência líquida positiva e o controle realiza a partida da rotação dos cilindros.

Em seguida o algoritmo de controle faz a leitura da potência líquida $P_{k}$ e calcula a diferença $\Delta \mathrm{P}$ entre a potência líquida atual $\mathrm{P}_{\mathrm{k}}$ e a prévia $\mathrm{P}_{\mathrm{k}-1}$. É também calculada a diferença de tensão de $\Delta \mathrm{V}_{\text {ref }}$ do "PWM Servo" resultado da diferença entre $\mathrm{V}_{\text {ref }(\mathrm{k})}$ (atual) e $\mathrm{V}_{\text {ref (k-1) }}$ (prévia). Se a $\mathrm{P}_{\mathrm{k}}$ (potência líquida atual) é negativa o algoritmo de controle utiliza um incremento $\Delta \mathrm{V}_{\text {fixo }}$ (passo fixo) para a tensão de referência $V_{\text {ref }}$. 
Se $\mathrm{P}_{\mathrm{k}}$ permanecer negativa por um longo período pré-estabelecido $\mathrm{t}_{\mathrm{w}}$, o acionamento dos cilindros é paralisado ajustando-se $\mathrm{V}_{\text {ref }}=0$ para também evitar desperdício de energia. Se $\mathrm{P}_{\mathrm{k}}$ é positiva, o algoritmo HCC (passo adaptativo) incrementa e decrementa $\mathrm{V}_{\text {ref }}$ com passo $\Delta \mathrm{V}_{\text {adap }}$ rastreando o ponto de potência máxima.

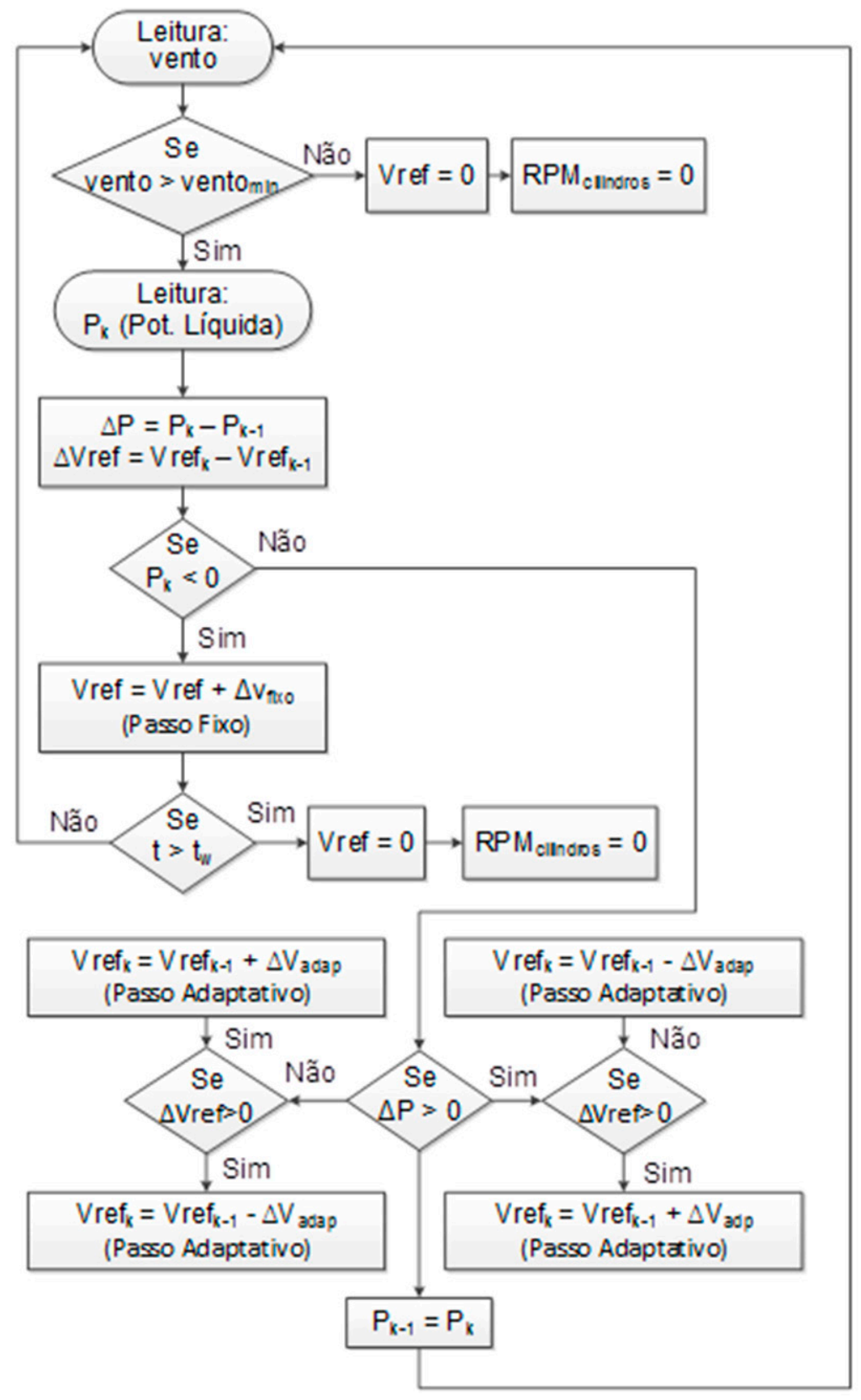

Figura 10 Fluxograma do algoritmo de controle da rotação dos cilindros. 


\subsection{Sistema de acionamento e controle da turbina Magnus proposto}

Na Figura 11 apresenta-se o diagrama de acionamento e controle dos cilindros girantes utilizado nos dois protótipos de turbinas Magnus apresentados anteriormente.

Faz-se a leitura do anemômetro (tensão $V_{c c}$ ou frequência) e da potência elétrica líquida $\mathrm{P}_{\text {liq }}$ entregue à rede elétrica.

$\mathrm{O}$ algoritmo HCC de MPPT executado no microcontrolador realiza o rastreamento da máxima potência líquida. O sinal de saída do microcontrolador é um PMW (Pulse Width Modulation), que é convertido em um sinal de nível CC e de nível CC para um sinal de onda quadrada de frequência controlada. Esse sinal de frequência passa através do anel coletor especial e serve de sinal de referência de rotação dos cilindros para o servo drive.

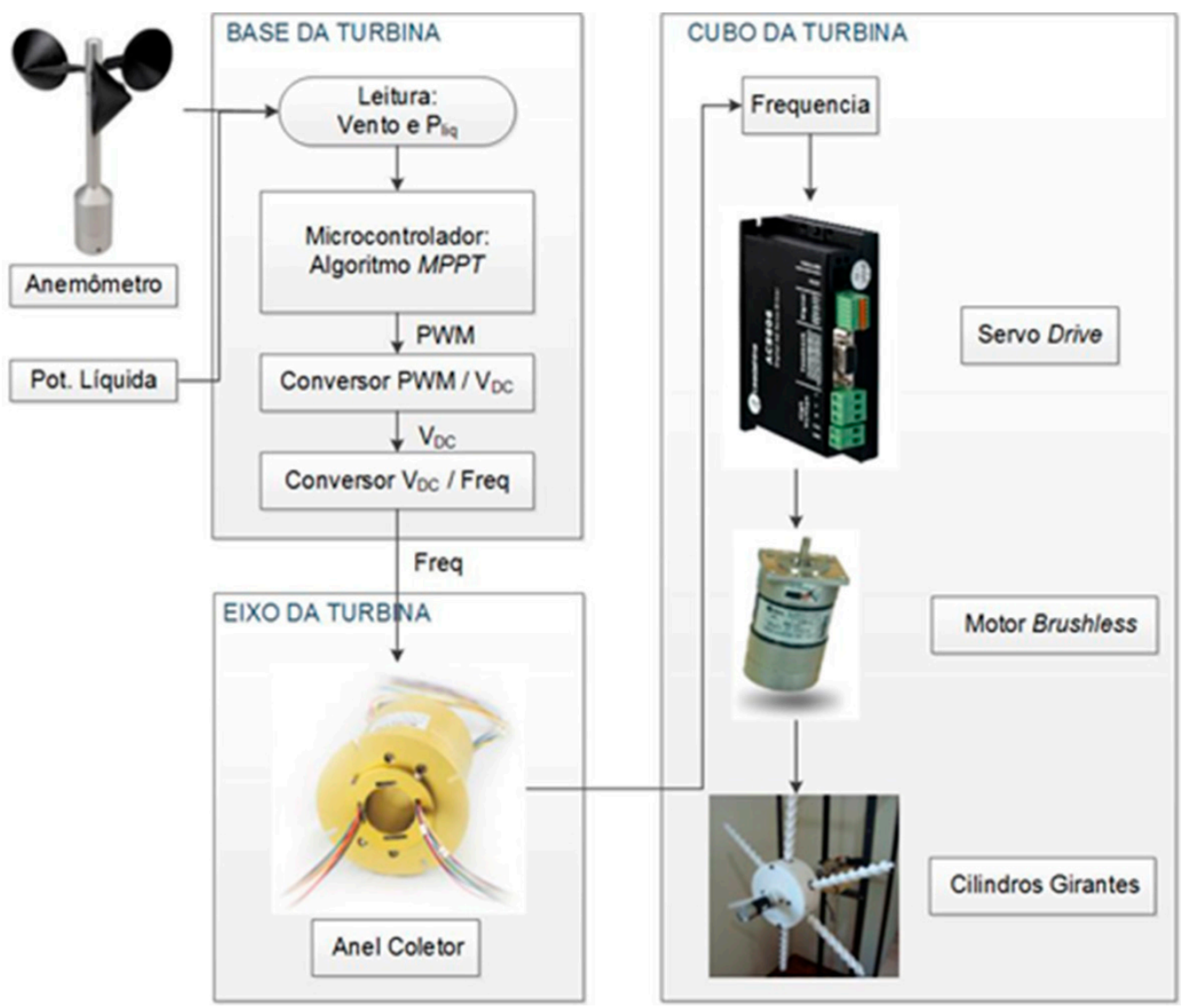

Figura 11 Sistema de acionamento e controle dos cilindros. 


\section{MODELO DINÂMICO DAS TURBINAS EÓLICAS CONVENCIONAIS}

Os fabricantes de turbinas eólicas convencionais de eixo horizontal não costumam disponibilizar prontamente as informações sobre o coeficiente de potência de seus produtos. Existem várias aproximações numéricas desenvolvidas em computador para o coeficiente de potência $\mathrm{C}_{\mathrm{p}}$ (MONROY, 2006), (MANXELL, 2009), (MUHANDO, 2008), (ZOBAA, 2011). A Equação (9), juntamente com Equação (10), é uma aproximação de uma função não linear para as turbinas eólicas convencionais de 3 pás.

$$
\begin{gathered}
\mathrm{C}_{\mathrm{P}}(\lambda, \beta)=\mathrm{C}_{1}\left(\mathrm{C}_{2} \lambda_{\mathrm{i}}-\mathrm{C}_{3} \beta-\mathrm{C}_{4}\right) \mathrm{e}^{-\mathrm{C} 5 \lambda_{\mathrm{i}}}+\mathrm{C}_{6} \lambda \\
\lambda_{\mathrm{i}}=\left(\frac{1}{\lambda+0,08 \beta}-\frac{0,035}{\beta^{3}+1}\right)
\end{gathered}
$$

$\mathrm{Na}$ Tabela 1, constam os valores dos coeficientes $\mathrm{C}_{1}, \mathrm{C}_{2}, \mathrm{C}_{3}, \mathrm{C}_{4}, \mathrm{C}_{5}$ e $\mathrm{C}_{6}$ para uma turbina eólica convencional com três pás (SONG, 2003), sendo que o $\beta$ é o ângulo das pás em relação à direção do vento:

Tabela 1 Coeficientes de $C_{p}$ de turbine eólica convencional de 3 pás

\begin{tabular}{cccccc}
\hline$C_{1}$ & $C_{2}$ & $C_{3}$ & $C_{4}$ & $C_{5}$ & $C_{6}$ \\
0,5176 & 116 & 0,4 & 5 & 21 & 0,0068 \\
\hline
\end{tabular}

A Equação (11) é a relação entre a velocidade tangencial da extremidade das pás e a velocidade do vento conhecido como TSR, similar ao da turbina Magnus:

$$
\lambda_{\mathrm{i}}=\frac{\omega_{\mathrm{t}} \cdot \mathrm{R}}{\mathrm{U}_{\infty}}
$$

O coeficiente de potência $C_{p}$ varia em função de $\lambda$ (TSR), ver Equação (9), onde $\omega_{\mathrm{t}}$ é a velocidade de rotação da turbina em [rad/s] e $\mathrm{U}_{\infty}$ a velocidade do vento laminar em $[\mathrm{m} / \mathrm{s}]$. $\mathrm{O} \mathrm{C}_{\mathrm{p}}$ representa o desempenho da turbina, ou seja, o quanto de potência ele consegue capturar do vento.

$\mathrm{Na}$ Figura 12, apresenta-se a curva do coeficiente de potência $\mathrm{C}_{\mathrm{p}}$ em função da velocidade relativa da turbina $\lambda$ para as turbinas modernas de três pás. O máximo valor de $C_{p}$ é apenas 0,47 com valor de $\lambda$ igual a 7 , o que consiste em um valor menor que o limite de Betz, devido às perdas por arrasto, turbulências nas extremidades das pás e perdas por stall (BURTON et al., 2011). 


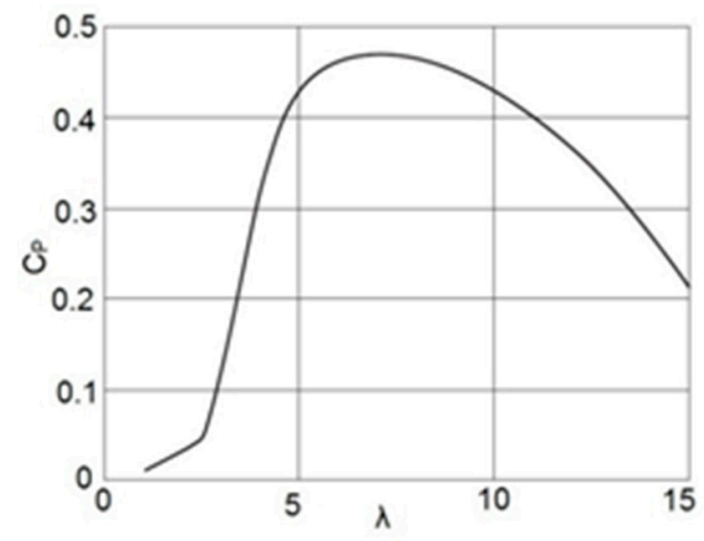

Figura $12 C_{p}$ em função de $\lambda(T S R)$ de modernas turbinas de 3 pás.

Fonte: Burton et al. (2011).

As curvas de coeficiente de potência $\mathrm{C}_{\mathrm{p}}$ apresentadas na Figura 13 foram obtidas através da Equação (9), utilizando-se os valores dos coeficientes da Tabela 1 referentes às turbinas eólicas horizontais convencionais de três pás.

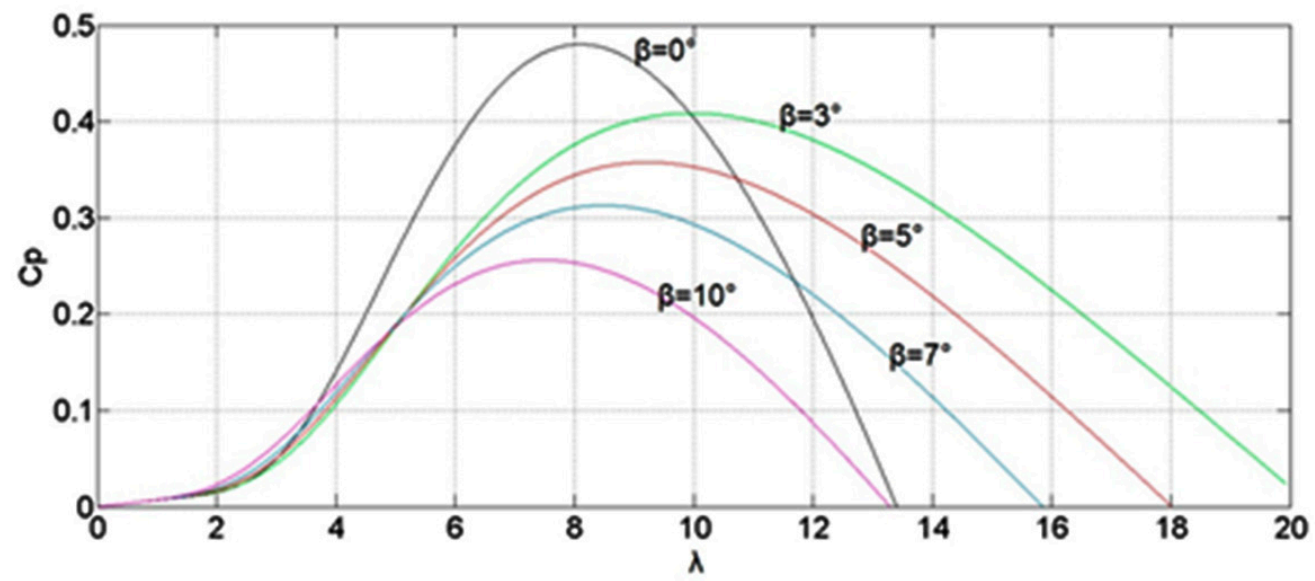

Figura 13 Variação de $C_{p}$ com $\beta$ e $\lambda$ de turbinas eólicas de três pás.

\subsection{Potências calculadas das turbinas Magnus e convencional}

Nesta seção, apresentam-se as curvas de potências mecânicas teóricas produzidas pelas turbinas eólicas convencionais e Magnus de acordo com a Equação (3) 
para várias velocidades de ventos possibilitando um comparativo. As potências mecânicas geradas pelas turbinas dependem do seu coeficiente de potência $\mathrm{C}_{\mathrm{p}}$. $\mathrm{O}$ $\mathrm{C}_{\mathrm{p}}$ da turbina convencional adotado nos cálculos analíticos é de acordo com a Equação (9) e o $C_{p}$ da turbina Magnus de acordo com a Equação (4).

As curvas de potência da turbina convencional nas figuras desta seção estão em função do TSR e as curvas de potência da turbina Magnus estão em função de $\lambda_{2}$ (velocidade relativa dos cilindros girantes). Essas curvas não levam em consideração a potência elétrica necessária para o acionamento elétrico dos cilindros girantes no caso das figuras da potência da turbina Magnus.

No sistema de acionamento dos cilindros dos dois protótipos apresentados pode-se considerar que a potência elétrica consumida $\mathrm{P}_{\text {cons }}$ pelo motor brushless é em torno de 37,5 W (25 V 1,5 A). Essas curvas que serão apresentadas a respeito das potências são cálculos analíticos e podem não expressar fielmente o comportamento real das turbinas.

$\mathrm{Na}$ Figura 14(a), apresentam-se as curvas de potências teóricas geradas em função da velocidade relativa dos cilindros $\lambda_{2}$ para uma turbina Magnus conforme dados da Tabela 2.

Tabela 2 Parâmetros da Turbina Magnus $R_{1}=1,15 \mathrm{~m} \mathrm{e} R_{2}=0,025 \mathrm{~m}$

\begin{tabular}{ccc}
\hline Parâmetro & Descriç̃̃o & Valor \\
\hline$N$ & Número de cilindros & 6 \\
$R_{1}[\mathrm{~m}]$ & Raio da turbina & 1,15 \\
$R_{2}[\mathrm{~m}]$ & Raio dos cilindros & 0,025 \\
Vento $1[\mathrm{~m} / \mathrm{s}]$ & Faixa de maior incidência & 3,5 a 7,5 \\
Vento $2[\mathrm{~m} / \mathrm{s}]$ & Faixa de baixa incidênica & 7,5 a 12,5 \\
Vento $3[\mathrm{~m} / \mathrm{s}]$ & Faixa de rara incidência & 12,5 a 40 \\
$\lambda_{1}$ & Tip Speed Ratio (TSR) & 1,0 \\
$\Omega_{1}[R P M]$ & Rotação da turbine & 0 a 48 \\
$\lambda_{2}$ & Velocidade relativa do cilindro & 0 a 1 \\
$\Omega_{2}[$ RPM] & Rotação dos cilindros & 0 a 2865 \\
\hline
\end{tabular}

Na Figura 14(b), apresentam-se as curvas de potências teóricas geradas em função da velocidade relativa da turbina $\lambda_{1}$ (TSR) para uma turbina convencional de mesmo raio da turbina Magnus. A faixa de velocidade do vento considerada na literatura de maior incidência. 

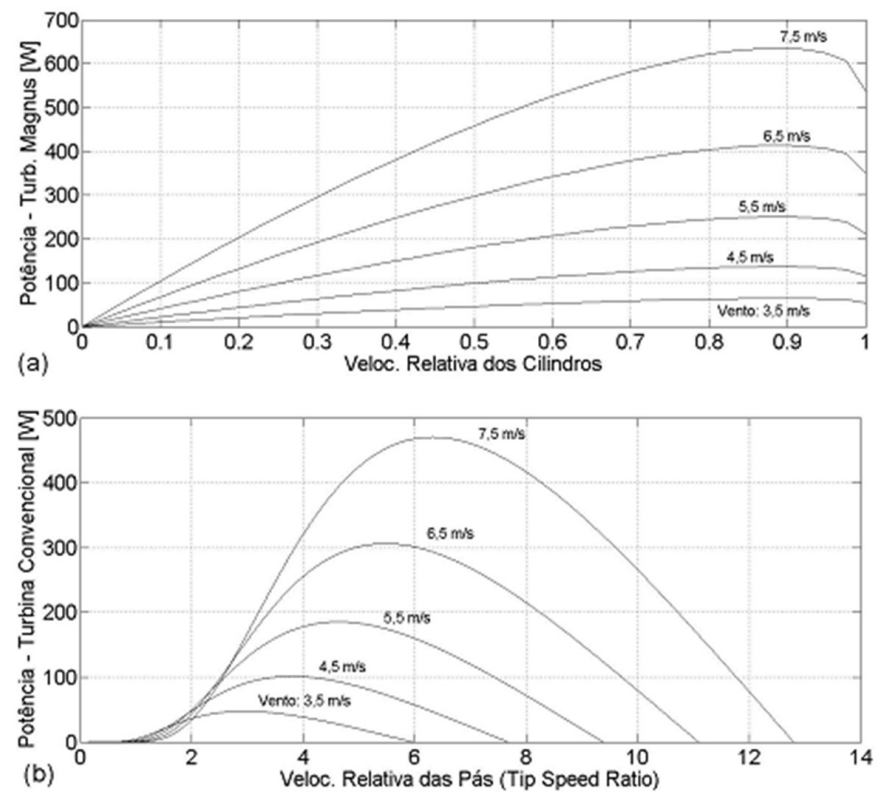

Figura 14 Potências mecânicas calculadas para ventos de maiores incidências.

Nas Figuras 15(a) e (b), apresentam-se as curvas das potências teóricas geradas. A faixa de velocidades dos ventos é considerada de baixa incidência.
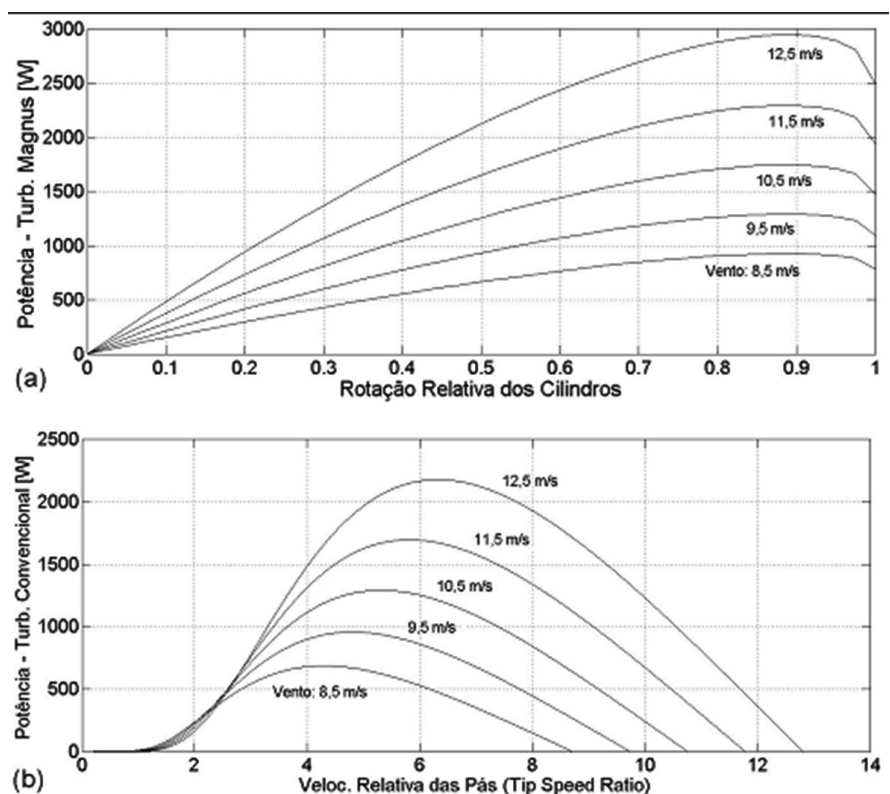

(a) Turbina Magnus. (b) Turbina Convencional.

Figura 15 Potências calculadas na faixa de ventos de baixas incidências. 
A Figura 16 apresenta as curvas de potências mecânicas teóricas geradas turbinas Magnus para uma velocidade de vento de $7,5 \mathrm{~m} / \mathrm{s}$ para 5 comprimentos de cilindros diferentes com raio de $R_{2}=0,025 \mathrm{~m}$.

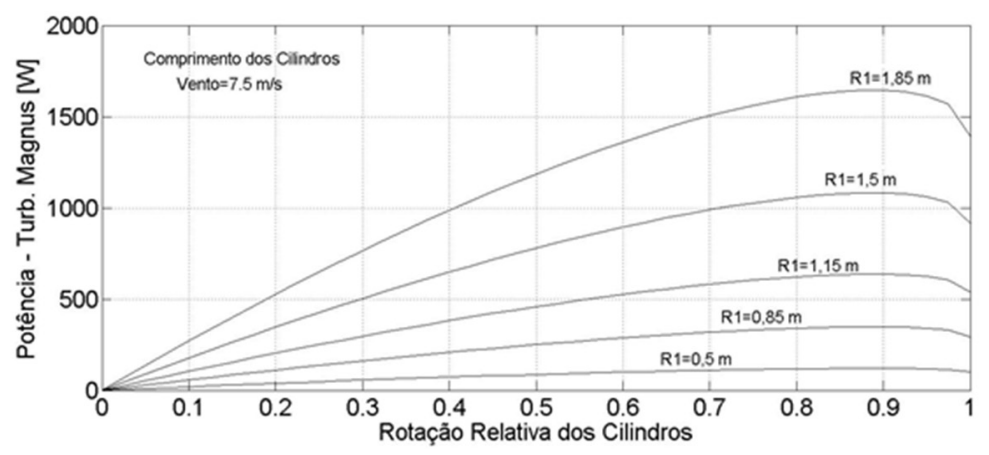

Figura 16 Potência calculada para vento de 7,5 m/s. Variações no diâmetro da turbina Magnus.

\section{CONCLUSÕES}

Duas concepções de turbina eólica de efeito Magnus foram apresentadas, um protótipo de $1 \mathrm{~m}$ de diâmetro fabricado em poliacetal, em que cada um dos cilindros está preso ao rotor por dois mancais internos ao cubo. O outro protótipo apresentado de $3 \mathrm{~m}$ de diâmetro possui eixos tubulares internos de alumínio e tubos de PVC externos girantes com barbatanas em espiral de polietileno.

Baseado nos resultados experimentais em testes em túnel de vento que foram realizados no IFSC câmpus Chapecó com os protótipos apresentados, pode-se afirmar que as barbatanas em espiral provocam um efeito aerodinâmico de aumentar a força de sustentação decorrente do efeito Magnus em relação a cilindros lisos.

O sistema de controle e o algoritmo de MPPT apresentados foram testados e funcionam de forma eficaz tanto para o controle da rotação dos cilindros como para o controle da razão cíclica de um conversor estático CC/CC (boost).

O modelo matemático do coeficiente de potência $\mathrm{C}_{\mathrm{p}}$ da turbina eólica de efeito Magnus melhorada com resultados experimentais em túnel de vento de (LUO, 2010) representada pela Equação (4) foi aplicada na equação de potência mecânica de turbinas eólicas, Equação (3). Com essa equação de potência obtida, geraram-se as curvas de potência apresentadas de forma comparativa com as curvas de potência de uma turbina eólica convencional de três pás representadas pelas Equações (9) e (10). Nesta comparação entre potências teóricas geradas, levando-se em consideração a potência elétrica consumida por um motor brushless (sem escovas), pode-se verificar o desempenho teórico superior da turbina Magnus em relação às turbinas eólicas convencionais para este diâmetro de turbinas. Pode-se 
constatar que o aumento do comprimento dos cilindros implica no aumento da potência mecânica da turbina Magnus.

\section{AGRADECIMENTOS}

Os autores agradecem ao CNPq (Conselho Nacional de Desenvolvimento Científico e Tecnológico) pelo financiamento das pesquisas apresentadas neste trabalho.

\section{REFERÊNCIAS}

BURTON, T. et al. Wind energy handbook. 2. ed. New York: John Wiley \& Sons, 2011. p. 4-127.

BYCHKOV, N.; DOVGAL, A.; KOZLOV, V. Magnus Wind Turbine as an Alternative to the Blade Ones. Journal of Physics: Conference Series 75, 2007.

. Parametric optimization of the magnus wind turbine. International Conference on Methods of Aerophysical Research, ICMAR 2008.

BYCHKOV, N. M. Magnus Wind Turbine, Calculated Characteristics of the Wind Wheel. Thermophysics and Aeromechanics, v. 15, n. 2, 2008.

DUAN, R.; LIN, C.; WAI, R. Maximum-power-extraction algorithm for grid-connected PMSG wind generation system. IEEE, 2006.

HAN, K; CHEN, G. A novel control strategy of wind turbine MPPT implementation for direct-drive PMSG wind generation imitation platform. IEEE, 2009.

IMAI, Y; KATO, C. Research of cylinder with spiral fin for Magnus wind turbine. Seisan Kenkyu. Institute of Industrial Science - University of Tokyo, 2010.

JINBO, M. et al. MPPT of Magnus wind system with DC servo drive for the cylinders and boost converter. Journal of Wind Energy, 2015.

LEADSHINE. Servo drive and brushless motor. Disponível em: <www.ledshine.com>. Acesso em: 26 ago. 2016.

LUO; D.; HUANG, D; WU, G. Analytical solution on Magnus wind turbine power performance based on the blade element momentum theory. Journal of Renewable and Sustainable Energy, v. 3, 2011.

MANXELL, J.F.; MACGOVAN, J.G.; ROGERS, A.L. Wind energy explained: theory, design and application. 2. ed. New York: John Wiley \& Sons, 2009. p. 100.

MECARO LTDA. Fabricante de turbinas eólicas Magnus. 2007. Disponível em: <http:// www.mecaro.jp/eng/>. Acesso em: 26 jul. 2016.

MOFLON. Slip ring. Disponível em: <www.moflon.com>. Acesso em: 26 ago. 2016.

MONROY, A.; ICAZA, L. A. Real-time identification of wind turbine rotor power coefficient. Proceedings of the $45^{\text {th }}$ IEEE Conference on Decision \& Control, 2006.

MUHANDO, B. E. Modeling-based design of intelligent control paradigms for modern wind generating systems. Tesis. University of the Ryukyus, Japan, 2008. p. 20. 
MUNSON, B.R. et al. Fundamentals of fluid mechanics. 8. ed. New York: John Wiley \& Sons, 2009. p. 304.

MURAKAMI, N.; ITO, J. Magnus type wind power generator. United States Patent. US7.504.740 B2. Mar., 17, 2009.

RIBEIRO, C. G. et al. Maximum power point tracking for magnus wind turbines. IEEE, 2013.

RUSSEL, S.; NORVIG, P. Artificial intelligence: a modern approach. 3. ed. New York: Pearson, 2010. p. 120-125.

SIMÕES, M. G.; FARRET F. A. Renewable energy systems: design and analysis with induction generators. New York: CRC Press, 2004. p. 197-208.

SLOOTWEG, J.G. et al. General model for representing variable speed wind turbines in power system dynamics simulations. IEEE Trans. Power Systems, v.18, n. 1, 2003.

SUN, X. et al. A three-dimensional numerical study of the Magnus wind turbine with different blade shapes. Journal of Renewable and Sustainable Energy, v. 4, 2012.

VAUGHN, N. Wind energy: renewable energy and the environment. New York: CRC Press, 2009. p. 05-93.

WHITE, F. M. Fluid mechanics. 4. ed. New York: McGraw-Hill. 2012. p. 509-518.

YANG, X.; GONG, X.; QIAO, W. Mechanical sensorless maximum power tracking control for direct-drive msg wind turbines. IEEE, 2010.

ZOBAA, A. F.; BANSAL, R. Handbook of renewable energy technology. New York: World Scientific, 2011. p. 37.

YOUTUBE. Magnus Turbine Prototype II. Disponível em: <https://youtu.be/ W9LuwX6mWFg>. Acesso em: 26 ago. 2015. 